\title{
Persuasive interface: Designing for the WWW
}

\author{
Dormann, Claire
}

Published in:

Professional Communication Conference, 1997. IPCC '97 Proceedings. Crossroads in Communication., 1997 IEEE International

Link to article, DOI:

10.1109/IPCC.1997.637063

Publication date:

1997

Document Version

Publisher's PDF, also known as Version of record

Link back to DTU Orbit

Citation (APA):

Dormann, C. (1997). Persuasive interface: Designing for the WWW. In Professional Communication Conference, 1997. IPCC '97 Proceedings. Crossroads in Communication., 1997 IEEE International (pp. 345-353). IEEE. https://doi.org/10.1109/IPCC.1997.637063

\section{General rights}

Copyright and moral rights for the publications made accessible in the public portal are retained by the authors and/or other copyright owners and it is a condition of accessing publications that users recognise and abide by the legal requirements associated with these rights.

- Users may download and print one copy of any publication from the public portal for the purpose of private study or research.

- You may not further distribute the material or use it for any profit-making activity or commercial gain

- You may freely distribute the URL identifying the publication in the public portal

If you believe that this document breaches copyright please contact us providing details, and we will remove access to the work immediately and investigate your claim 


\title{
Persuasive Interface: Designing for the WWW
}

\author{
Claire Dormann \\ Center for Tele-Information, DTU \\ Building 371, DK-2800 Lyngby \\ claire@cti.dtu.dk
}

The design of web documents is approached from the perspective of visual rhetoric and important aspects of persuasive discourses (e.g. the role of emotion) will be presented in connection with the world wide web (WWW). In this paper, the origin of the rhetorical figures is first briefly outlined. Then, a few rhetorical figures such as metaphor, metonymy or visual pun are described and illustrated in relation with web pages. The figures pertain to every aspect of web design, images and concepts used to convey meaning, and the detailed visual characteristics of all web components. Subsequently, their application to the design of web pages is discussed. The rhetorical perspective suggests manners in which to express the most efficient statement. Rhetorical figures are techniques that are employed to enhance documents or to find original solutions to design problems. The concern here is to make web authors aware of rhetorical figures and to lay the foundation of a rhetoric framework grounded in web design.

\section{Introduction}

The growth of WWW into all aspects of society means that web documents have to be used by non-professional computer users, more familiar with the conventions of massmedia than the conventions for graphical user interfaces. In the search of good design guidelines and practices, applying a perspective such as rhetoric might prove more effective than applying a purely technological perspective. Rhetoric, generally defined as the study of the means of persuasion, has provided tools (i.e., rhetorical figures) that can assist authors in their design tasks.

In this paper, we will introduce rhetorical figures in relation to the WWW and show the relevance of visual rhetoric to the design task. While doing so, we will review important aspects of web design e.g. as a time-based component and its consequence for the figure design. We will also highlight the role of emotion and its importance in communication.

\section{Rhetoric theory}

Rhetoric originated from the functional organisation of verbal discourse. Its object is eloquence (or proficiency) defined as effective speech designed to influence and to convince others. Traditionally, there is a choice of three strategies of appeal which can be adopted to persuade others: the ethical, the emotional, and the rational. In the classical system, rhetoric was divided into four phases reflecting the different stages that speakers ideally go through: invention, disposition, elocution, and action. Elocution as the form in which ideas and arguments are expressed, is of particular interest. It covers the study of figures of speech or rhetorical figures. These figures are classified according to their relation to the discourse. Distinctions involve the difference between syntactic and 
semantic figures or are based on the types of operation involved in the figure creation (e.g. similarity or substitution).

Some of these textual figures have been translated and discussed in relation to visual discourse in fields such as: graphic design (e.g., Ehses, 1989), user interfaces (UI) (e.g., Marcus, 1993), and cinema (e.g., William, 1981). Web design developed initially from the work on user interface design (especially in relation to UI metaphors). However web design is situated at the crossroads of different disciplines, and thus perspectives from advertisement and design might prove more appropriate than a pure technological approach.

As UI metaphors are often discussed in relation with web design, we will first situate this figure in the context of rhetorical figures taxonomies. Then we will present other rhetorical figures and show their relevance to the design of persuasive messages. As in the rhetoric tradition, a great emphasis is put into the integration of the affective and rationale element of communication, we will specifically discuss this topic.

\subsection{UI metaphor and the rhetorical system.}

Users are often confused about the nature of metaphorical relations in interfaces (Madsen, 1994). We might find some answers by looking at rhetoric theories and more specifically at rhetorical figures taxonomies.

Users often interpret metaphor as simile or analogies. Analogies employ direct mappings between the source and target thereby encouraging the direct transferal of existing knowledge to a novel situation. Presenting a software artifact as a direct analogue to a physical object almost always imposes unnecessary visual and conceptual restrictions on the design (Smyth and Knott, 1994). The simile draws a limited comparison between one thing and another ("as fierce as a tiger" -- fieriness is the sole quality compared). However, a metaphor is defined as one thing described as something else. Metaphors draw incomplete parallels between unlike things, emphasising some qualities and suppressing others. The power of the metaphor lies in its ability to be extended in flexible and creative ways which do not precisely mirror the source of the metaphor. Users should be encouraged to explore the links between the source and the target, thereby clarifying those features of the source that are appropriate in the current context of use.

\subsection{Metaphor or catachresis}

We should also look more closely at the role of metaphor in user interface design. The traditional physical media is a natural starting point for metaphoric representations. Examples for the web include libraries with doors, help desk rooms, collections and shelves or the city of knowledge with gates, streets, buildings and landmarks for interface function and representation (Shneiderman, 1997).

There exists some voluntary alterations to alleviate insufficiencies of vocabulary: in a situation where a word does not exist, a new one has to be made up, or the meaning of an old word has to be shifted. Fontanier (1982) defines the new word mechanism into the rhetorical figures as catachresis. Catachresis refers to a situation in which a sign, already 
assigned to a first idea, is assigned to a new idea, this latter idea having no sign at all or no proper sign in the language.

In UI, metaphors might play a very specific role, quite distinctive from other roles of metaphor (see section 4.4), and as a matter of fact, also different from the utilisation of other rhetorical figures in design (including the WWW).

\section{Rhetorical figures within the WWW}

So far, we have focused on metaphor, however metaphor and similar devices are only a small part of the catalogue of rhetorical figures. In the textual word, Molinie (1992) categorises 140 figures. Here, we are going to discuss some of these figures in relation to the WWW.

\subsection{Metonymy and synecdoche}

Metonymy and synecdoche are frequently found in web pages. Synecdoche is defined as the substitution of a more inclusive term by one that is less inclusive; the part for the whole. Metonymy is defined as the substitution of terms suggesting an actual relationship, the substitution can be of a causal, spatial or chronological nature). This device seems a natural starting point for the design of icons such as those representing site topics. The different metonymic relations (e.g. container for contain, producer for product, etc.) can be explored in order to find the best relationship. These figures are employed as much for interface elements as for the site's visual content. Examples include a graduation cap for a link on careers and jobs or a photo of curator head inbetween dinosaurs jaws (for a science museum site). The use of the jaws in this way (instead of the whole dinosaur body) is quite powerful.

\subsection{Visual pun}

Visual pun can be used to express more information in a small design (useful for icon), to reinforce the identity of a web site (or elements) or in more playful manner. A photograph's objective which contains a small picture of homeless men gives access to a site containing such photos (Voyager, 1997). A typical example is a header where some letters have been replaced by objects reflecting the title content. In a film noir site navigation arrows have been replaced by pistols. For another such site, the designer has used a bullet hole for bullet point. Using visual pun in the design of bullet points seems quite frequent. Another example is given by Levine (1996) where different picture of bagels are used for a bagel list. Such designs help guide users' navigation and facilitate their understanding of Web information.

\subsection{Hyperbole and personification}

Hyperbole is the use of exaggeration for emphasis and is used to make an object more prominent. Examples of hyperbole are found in pictures of film noir sites where the size of the murder weapons (e.g., knife or gun) are greatly enhanced. Moreover, the angle and part of the weapon emphased are always chosen so as to give the most dramatic impact. Another web site on thrillers contains an opposite example of hyperbole (i.e. tapinose, exaggeration by minimising) where a small human figure is placed in the middle of huge blow-up of a metallic and glass structure. 
Personification is defined as a comparison whereby human qualities are assigned to inanimate object, for example, by adding legs, arms or a face to an object is a very common figure in advertising where almost any objects are 'humanised' and animated. This has transferred to web site design. Curiously, one instantiation of this figure (e.g. a personified computer) found in cartoons or animated gif seemed popular and is used for humoristic purpose. Such instances were also found in computer books or manuals (DTI, 1990). Other examples of personification include a light bulb for a young engineer graduate home page and a smiling earth for Hot Wired website (Hot Wired,1997).

\subsection{Accumulation}

Figures of accumulation such as amplification (i.e., the expansion of a topic through the assemblage of relevant particulars) is often used in advertising to reflect the various products sold in a shop. In web design, it can correspond to the site flavor. Occurrences of this figure are found in the home page image map. In Microsoft explorer, a small imagemap is composed of two transparent shots of a pianist on a background of music sheets. The first shot represents a small top view of a pianist and the second a close-up on the hands and keyboard.

In the virtual hospital (1997), an image map consists in a collage of different pictures showing different close-up of doctors or of doctors and patients (i.e. symbolising caring attitudes).

Accumulation can be based on figures like metonymy and synecdoche. This figure has also been found in gif animation. When entering a mountain bike site, an animated gif placed besides the header consists of a cycling series of three images. First a cyclist head with a helmet is shown (nice touch on safety), then the handle bar and cyclist hand, and last the bike body. The choice of pictures could represents different site aspects: e.g., safety, mechanics and trademarks.

\subsection{Repetition}

Anaphora is the duplication of a word or a group of words, it is the most elementary figure of repetition. The most well-know visual examples of anaphora is Marilyne Monroe by Andy Warhols. An image map linking to 'car point' uses a repetition of car body. Similar cases has been found in different web sites. The effect of repetition can be established using virtually any design elements.

Another interpretation of repetition is found in an photo (multiple reflections of a couple in mirror) coming from a famous scene from the "Lady from Shanghai", the hall of mirrors is symbolic of the situation in which Welles (the heroine) find herself, nothing is what is appears, everything is an illusion.

Repetition is quite frequent in background where the same element is repeated across the page giving a tile effect. Alternatives include repetition of groups of elements such as planets for this computer services site (InfoMedia,1997). Variations are obtained by changing the element colour or size.

The repetition of elements throughout the web pages reinforces the nature of the product while enhancing the continuity of the document as a whole. Repetition facilitates communication. The repetition of elements across the site produces an effect similar 
visual theme used in cinema. Staircases are a frequent visual theme in thrillers which are carried across the film.

\subsection{Other figures}

There are numerous other textual figures which can be translated visually and which can be used in web design. In anacoluthon the exchange of elements results in the breaking of syntactic rules. The visual equivalent is an impossible image such as a wardrobe opening to a new world (Durand, 1970). City and rooms are often used to present information. We could design a cascade flowing out of wardrobe, sand and crabs dripping out of a chest as anchors for maritime words of a travel or environmental agencies.

Gradation consists of ordering words by length thus a variation of an object size is a very pure form of graduation Antithesis is the juxtaposition of contrasting ideas, often in parallel structure. Visually, an opposition of black and white and coloured images can be created. Litote is the deliberate use of understatement, not to deceive someone but to enhance the impressiveness of what we say. Visually it can be expressed by very small images or text figuring in a almost empty web page.

\subsection{Discussion}

As we have seen, rhetorical techniques pertain to every design aspect of the web document including the interaction techniques, images and concepts used to convey function and meaning on the computer screen. In a word to the detailed visual characteristics of every component of a page and functional sequences of interactions over time that produce the characteristic "look and feel" of WWW documents. Although we have mainly discussed rhetorical figures in relation to the image, we should look briefly at their utilisation within animation and video as they are becoming an increasingly important part of web design.

Figures can take place across a scene, or a sequence. In Battleship Potemkin, by Eisenstein, a close-up shot the pince-nez of the ship's doctor is shown dangling on a rope to convey that the doctor has been thrown overboard. The metonymy is based on the fact that the glasses and the doctor had been continuously associated during the film. Associated details can be compressed within the limits of the frame to present a statement of extraordinary richness. Metonymy is a kind of cinematic shorthand. That might prove an extremely important technique for web design as important constraints in space and time exit.

Besides, video and animation give rise to new interpretation of figures such as anticipation or anastrophe. Anticipation is a way of exaggerating a preliminary action in order to give the audience a cue about the main action to follow. It would translate directly to web animation. Anastrophe is the inversion of a natural or usual word order. A typical visual example is a deconstructed object reconstituting itself in seconds such a computer reassembling itself (e.g. as animated gif) for a computer hardware site.

\section{Toward persuasive interface}

We have illustrated the utilisation of rhetorical figures. We are now going to look more closely at the role of these figures in relation to design, stressing two specific aspects of 
rhetoric discourse: emotion and connotation and their importance to web discourse. As we have seen, the role of rhetorical figures in UI is similar to "word creation" or is turned toward the rational aspect (or "Logo") of classical rhetoric. However rhetoric integrates the affective and rational elements of communication into the same whole. Thus, the role of emotion needs to be explored in a more systematic way.

\subsection{Importance of emotion}

In eighteen-century England, the Augustan culture appealed pre-eminently to reason and the more imaginative forms of emotion and emotional expression were suspect. It might be than within the traditional computer culture a similar phenomenon has occured, however such is not the case with other media.

As pointed out by Disney animators (Frank, 1981), the key concept in cartoon animation is that communication is achieved through audience involvement. Disney animators start with something that the audience knows and likes. This can be either an idea or a character, chosen because it is familiar and appealing. The fact that speakers and writers deliberately play on the emotions of their audiences cannot be escaped, how we feel about an issue relates to our understanding of it. Nash (1982) demonstrates convincingly how the arousal of feeling involves the use of argument and repetition, and its through these means, together with associated stylistic patterns such as antithesis and rhythmic structures, that a powerful emotional interaction is established between persuader and audience.

An interesting example has been found on the home page of a virtual shopping site (Zapcity, 1997). The imagemap pictures a village square. To depict the building content metonymy has been used in the design of individual buildings (e.g. the top of phone company looks like a telephone receiver). Another building has round windows and an elongated red porch, reminding us of a friendly face (personification). This design conveys both a functional aspect (which building give access to which products) and an emotional intention (bright colour, friendly building) which required the utilisation of different rhetorical figures. The designer appeals to visitors and persuades them to visit the web site. Because web viewers are under no compulsion to start reading a home page, or continue their visit, an important function of rhetorical figures is to motivate potential viewers. As millions of web pages compete with each other, this might become increasingly important aspect of web design.

\subsection{Expressing emotion}

Emotion can be expressed through the use of humorist devices. Within the visual medium, visual puns occupy the same place as word games. The pun effect happens when a viewer becomes aware of the multiple meanings or associations. For a brief moment it stimulates the viewer's imagination by way of either memory or expectation. Another figure is personification (as we have seen) where all the techniques of visual comedy can be applied. There are other visual jokes based on different principles such as irony, satire, parody and caricature. Satire is based on criticism, parody depends on prior knowledge utilising the familiar in such way that we recognise both the original and the departure of the original. Parody relies upon a common frame of reference to be truly effective. 
The figures of irony may be included to support a brand's personality or call out to a particular target audience. Personification could serve as 'animated guide'. Satire and parody create a complicity of share experienced between author and audience. They could be employed for "cult web site" such as Star Trek. Furthermore for the science museum and the dinosaur section, a parody of Jurassic park could prove quite attractive for a younger public. Well-known personalities are often caricatured. The caricature of John Major (Net,1997) would have been quite appropriate for the English election web site.

\subsection{Audience motivation}

Another aspect of the rhetoric discourse is the importance of connotation in the communication. When a writer calls a ship 'a sail', the connotation indicates the desire (in the case of a synecdoche where a part is substituted for the whole) to designate the thing by means of a detail, or (in the case of metaphor) to designate it by means of a similarity. The same can be said for the gun designs (based on Hyperbole in film, noir), the telephone building (using metonymy in the shopping center) or of the shots selection in the pianist example. The use of the figure is far from neutral. It has been chosen for a reason and it is not equivalent to a more common expression. It can be added that what is untranslatable is the power of the figure to evoke an affective tone.

By using non ordinary expression the designer / authors create an element of surprise and stimulate viewer's interest. In fact most rhetoricians agree that the major effects of rhetorical figures are on attention and recall. Thus the positive effects on attention, liking and recall which these figures are expected to confer might prove a fundamental aspect of web design.

\subsection{Creative design}

The utilisation of the rhetorical tools to create a striking and unique message in order to persuade is seldom discussed in relation with web design. Web designers can not only emphasis a point by using synecdoche, but also with other techniques including hyperbole (e.g., by doubling the size of item), and accent (colouring some information in black and white pages). Crucial information will stand out on the web pages.

These devices can help guide viewers to the most significant information, by giving audience singposts to access information (e.g when using visual pun in the design of bullet points, the list content is immediatly apparent).

Using visual pun and anacoluthon, designers can take greater chances in communicating complex ideas. For at web site for public accountants (Api, 1997) portrays a computer with an adding machine scroll spilling out of the screen to join the keyboard. These devices connects the old world to the new electronic one. Metaphors are employed in education to explain complex notions (e.g. a picture of running water in a pipe to explain electricity). Rhetoric figures can give the message greater vitality and impact.

Rhetorical figures can also be used to create a thread across different web pages and to express more subtle relation in a web document. A specific relation can be created between a visual link and the new page or between a thumbnail and the full size picture. For example a thumbnail depicting a rhinoceros head and horn is given for a illustration 
picturing rhinoceros poaching, thereby creating a kind of antithesis between the full size picture and the thumbnail (Dormann, 1997).

These tools are used to stimulate viewers interest when creating unusual image (e.g., cascading wardrobe, api consultant, or as the tapinose example). By making an environment pleasant and using humorous devices we can overcome viewer resistance and persuade viewers to take action.

By choosing adequately, figures can render thoughts vividly concrete, helping designers to communicate with their audience clearly and effectively. Figures provide one of the best ways to strike that happy balance between the obvious and the obscure, so that the audience can grasp ideas promptly and thereby be disposed to accept the argument.

\subsection{Author}

We have seen how tools can be used to vary information, create striking messages and effectively appeal to the audience. However there are figures which are mainly defined in relation to the author of a message. Figures can elicit admiration for the eloquence or skill of the writer or designer. Thus, combining contradictory concepts in an oxymoron (i.e. the yoking of two terms which are ordinarily contradictory) to produce a startling effect may win a reputation of wit for an author. As the WWW technology can bring author and audience in contact, this might become an important aspect of web design and be particularly relevant for personal pages.

\section{Conclusion and future work}

We have discussed important aspect of web designs: the role of emotion the relation to author and audience, and the importance of connotation. We described how a wide variety of rhetorical figures could be integrated in the design of web site and we have tried to show how the persuasiveness of rhetorical figures can work. If a proposition can be expressed in a variety of ways, one of these ways will invariably be the most effective in swaying an audience. The rhetorical perspective suggests manners in which to express the most efficient statement. Rhetorical tools can be used as exploration tools that open choices and can assist designers in the creative process.

In doing so, we lay the foundation of a rhetoric framework grounded in web design. The proposed framework can be used to guide future research toward comparing and distinguishing the effects of different figures rather than focusing on individual figures. It will also be important to examine moderating variables that heighten or limit the efficiency of rhetorical figures. Moreover, we can expect that one kind of figure will be more effective than another in a particular respect or circumstance. The effect of rhetorical figures cannot be uniform across the web spectrum.

In this paper we have tried to make salient a largely unacknowledged and undifferentiated aspect of web design. A widespread use of rhetorical figures to serve as an effective mean to various circumstances for which web pages are produced will demand a wider range of visual figures than the ones discussed in this paper. Thus, the development of a taxonomy and examples situated in the field of Web design or linked to internet users is necessary. 


\section{References}

Durand, J. Rhétorique et image publicitaire. Communications, 15 (1970),70-95.

Dormann C. Retoriske figurer som udgangspunkt for Web-design. To be published in Design of multimedier. Ed Bo-Fibiger, denmark:Aalborg University, August 1997.

DTI, Department of Trade and Industry. A Guide to Usability. Milton Keynes: The Open University, 1990.

Ehses, H. Representing Macbeth: A Case Study in Visual Rhetorics. In Design Discourse, ed., V. Margolin, 187-199. London: Chicago University Press, 1989.

Fontanier, G. Les figures du discours. Paris: Editions du Seuil, 1982.

Frank, T. and O. Johnson. Disney, The Illusion of Life. New York. Abbeville Press, 1981.

Levine, R. (1996) Sun on the net: Guide to Web style, $<\mathrm{http}: / /$ www.sun.com/styleguide/>.

Madsen Haslskov K. A guide to metaphorical design. Communication of the ACM, 37: 12 (1994), 57-61.

Marcus, A. Human Communications Issues in Advanced User Interfaces. Communications of the ACM, 26: 4 (1993), 101-109.

Molinié, G. Dictionnaire de rhétorique. Paris: Librairie Générale Française, 1992.

Nash, W., Rhetoric: The Wit of Persuasion. Oxford: Blackwell, 1989.

Net. Special election. Net, 30, (Spring 1997), 53-67.

Shneiderman B. Designing Information-Abundant Websites: Issues and Recommendations. To be published in International Journal of Human-Computer Studies, July 1997, <http://kmi.open.ac.uk/ simonb/ijhcs-www/>.

Smyth, M. and R. P. Knott. The Role of Metaphor at the Human Computer Interface. Proceedings of OZCHI94 - Harmony through workingtogether, Melbourne, Australia, 1994, 287-291.

Williams, L. Dream rhetoric and film rhetoric: metaphor and metonymy in un Chien Andalou. Semiotica, 33:1/2 (1981), 87-103.

API AccountantS for the public interest, 1997, $<\mathrm{http}$ //accountingnet.com:80/asso/api/index.html $>$.

Hot wired, 1997 <http: //www.wired.com/wired/5.07/longboom/index.html>.

Virtual hospital, University of Iowa, Iowa, 1997. <http://vh.radiology.uiowa.edu/>

Voyager. Fragile Dwelling, $1997<\mathrm{http}: / / \mathrm{www}$.voyagerco.com/fragile/>.

Zapcity. Virtual commerce, 1997, <http://www.zapcity.dk/>.

InfoMedia. Computer Services, $1997<\mathrm{http}$ ://www.hjheld.comm/educate.html $>$. 